Maria Cecilia Costa'

Inês Lessa ${ }^{2}$

Solange Veloso Viana ${ }^{3}$

\section{Práticas Alimentares e Perfil Lipídico Indesejável em Trabalhadores Petroquímicos}

\author{
Eating Habits and Undesirable Lipidic \\ Profiles in Petrochemical Industry \\ Workers
}

No universo dos trabalhadores de uma indústria petroquímica de Camaçari, Bahia - Brasil foi realizado estudo de corte transversal, para medida da associação entre práticas alimentares e perfil lipídico indesejável. Os dados sobre hábitos alimentares e atividade física foram obtidos por questionário semi-estruturado e os dados secundários, clínico-laboratoriais e dietéticos, a partir da ficha clínica padronizada e da ficha do cardápio diário. Para análise, foram usadas as Prevalências (P), Intervalos de Confiança a 95\% (IC 95\%), Razões de Prevalência (RP) e regressão logística. A quota energética média, consumida diariamente, numa única refeição, na indústria, independentemente do turno de trabalho, correspondeu a $96,1 \%$ da quota energética, total, média diária, dos trabalhadores. A prevalência das práticas alimentares mais lipídicas foi de $63,9 \%$, IC (53,6; 74,2); de colesterol total indesejável de $49,4 \%$, IC $(38,6 ; 60,2)$ e a da não participação no Programa de Qualidade de Vida, de $60,2 \%$, IC $(49,7 ; 70,7)$. A RP entre práticas alimentares e perfil lipídico indesejável, de 1,43 , IC $(1,02 ; 2,01)$, com $p<0,05$.

Palavras-chaves Práticas Alimentares, Indústria Petroquímica, Lípidas Séricas, Alimentação Institucional.

A cross-sectional survey was conducted to assess the association between eating habits and undesirable lipidic profiles in the universe of petrochemical workers $(n=83)$ in Camaçari, Bahia, Brazil. The data concerning eating habits and physical activities were obtained using semi-structured questionnaires, while the secondary data, i.e. the clinical and laboratorial data were obtained using standardized clinical data registration forms and the daily menu records. Analysis input elements consisted of: Prevalences (P); Confidence Intervals up to 95\% (IC 95\%); Prevalence Quotient (RP) and Logistical Regressions. The average energetic quota ingested per day in a single meal within the industrial plant, independent of the work shift corresponded to $96.1 \%$ of the total average energetic quota for the workers. The prevalence of more lipidic eating habits was $63.9 \%$, IC (53.6; 74.2); undesirable cholesterol $49.4 \%$, IC $(38.6 ; 60.2)$ and non-participation in the Quality of Life Program was $60.2 \%$ (IC $(49,7 ; 70,7)$. The Prevalence Quotient (RP) between eating habits and undesirable lipid profile was $1.43, \mathrm{IC}(1.02 ; 2.01)$, with $\mathrm{p}<0.05$.

Keywords Eating Habits Practices, Petrochemical Industry, Lipidic Serum, Institutional Food Catering. 


\section{Introdução}

O processo de industrialização vem incorporando, de forma crescente, tecnologias com alto grau de automação ${ }^{23}$, com tendência de mudança do perfil de morbimortalidade dos trabalhadores $5,7,18$. Essa nova tendência, configura-se pela maior prevalência de agravos à saúde, representados, sobretudo, pelas doenças crônicodegenerativas, cuja relação de causalidade, com o trabalho, não é tão evidente, como o nexo que ocorre com as doenças, tipicamente, ocupacionais?. Essas doenças vêm contribuindo, para a redução do tempo de vida produtiva e ocupando posição de destaque, na composição das taxas de mortalidade, em diversos países, inclusive, nos países em desenvolvimento ${ }^{1,3,14}$.

Alguns autores $11,15,18,27$ sugerem, que a ocorrência de muitas das doenças crônicas, não transmissíveis, guardam estreita relação com as características qualitativas da dieta, sobretudo, a incorporação de maior quantidade de alimentos ricos em gorduras. Outros ${ }^{5,7}$ demonstram que esses novos hábitos são resultantes de ajustes às novas formas de consumo, em decorrência de novos padrões tecnológicos, além dos processos de urbanização e de migração. Essa questão assume importância singular, considerando que o monitoramento, permanente, da operação nas indústrias, transforma a produção e o fornecimento de refeições, numa exigência da própria tecnologia, para organizar 0 processo produtivo ${ }^{26}$. Nessas organizações, os trabalhadores consomem, pelo menos, uma refeição no local de trabalho, cujas diretrizes orientamse pelo Programa de Alimentação do Trabalhador - PAT ${ }^{4}$. O Programa propõe-se a resolver problemas relacionados à escassez de nutrientes, articulando saúde e produtividade, estabelecendo "quotas energéticas mínimas" para o trabalhador, indistintamente, sem qualquer avaliação, baseada em mínimos calóricos. As quotas resultam em comportamentos alimentares, que tanto podem contribuir para manter a saúde e o bem-estar, como para desencadear transtornos lipídicos e morbidades associadas ${ }^{11}$.

O reconhecimento de que as características da dieta podem exercer influência de- cisiva sobre o estado de saúde dos indivíduos levou a Organização Mundial de Saúde a estabelecer limites para o consumo de gorduras (de colesterol, de ácidos graxos saturados e poliinsaturados), de proteínas de origem animal e de glicídios, principalmente os monossacarídeos ${ }^{27}$. Considerando que as estratégias preventivas sobre fatores de risco ou de doenças no âmbito das indústrias, sejam eles adquiridos nela, ou em outro ambiente, são obrigatórias nos programas de saúde do trabalhador e que as dislipidemias, em grande parte, são resultantes do hábito alimentar, este estudo teve como objetivo, determinar a relação entre práticas alimentares, predominantemente na indústria, e a presença de perfil lipídico indesejável, dos seus trabalhadores.

\section{Metodologia}

Estudo de corte transversal, realizado em uma indústria química, do Pólo Petroquímico de Camaçari - BA, operando desde o início dos anos 90. abrange o universo dos 83 trabalhadores, com idade inferior a 50 anos, com vínculo empregatício, em atividade em 1998.

Características pessoais, socioambientais, do trabalho e comportamentais dos participantes, foram obtidas por questionário, auto-preenchido, previamente testado. As informações sobre colesterol total (CT), HDL colesterol (HDL-c), LDL colesterol (LDL-c), triglicérides $(\mathrm{Tg})$ e colesterol total/HDL colesterol (CT/HDL-c), foram obtidas dos prontuários médicos, no setor médico da indústria e, os dados sobre a composição da alimentação, para estimativa da quota energética, média diária, oferecida para consumo na indústria, referentes ao ano de 1998, foram identificados nas fichas dos cardápios diários, no serviço de nutrição da indústria. Esses cardápios foram, previamente analisados, a partir dos critérios estabelecidos pela política de alimentação da fábrica. Devido ao fato dos cardápios apresentarem o mesmo padrão calórico e a mesma freqüência das preparações, foi sorteado e analisado um mês de cardápio, para estimar a quota energética, média, oferecida para consumo na indústria. 


\section{Critérios e definições:}

1) Práticas alimentares foram classificadas em: a) mais lipídicas - quando na resposta ao questionário, o trabalhador mencionava como de consumo diário, alimentos e/ou preparações lipídicas, em pelo menos três refeições e, b) menos lipídicas - quando não era informado o consumo habitual diário de alimentos e/ou preparações lipídicas, nas três refeições consumidas - desjejum, almoço e jantar;

2) Perfil lipídico indesejável (PLI) - quando pelo menos uma das lípidas $-\mathrm{CT}$, HDL-c, LDL-c, Tg - ou o índice CT/HDL-c, apresentava o valor fora do limite recomendado24;

3) Participação no Programa de Qualidade de Vida (PQV*): a) não participante - quando o trabalhador negou a participação no programa, em qualquer período, desde a sua implantação e, b) participante - quando informou participação no programa, pelo menos uma vez.

4) Quotas energéticas, médias diárias recomendadas, foram definidas como: a) total (QET) - quando a quantidade de calorias, obtida pelo somatório das quotas energéticas médias, para cada atividade dos trabalhadores, praticada durante o dia, a partir da Taxa Metabólica Basal - TMB ${ }^{21}$, foi considerada como adequada às necessidades nutricionais médias da populaçãoalvo, após realizado o ajuste da quota energética média total diária, por faixa etária $^{22}$ e, b) Quota Energética Ocupacional (QEO) - quando a quantidade de calorias foi considerada restrita, para - desenvolvimento da atividade do trabaIhador na indústria, a partir da idade, do peso, da altura, do sexo e da atividade física ${ }^{2,22}$.

5) Quota Energética média, oferecida na Fábrica, para consumo diário, (QEF) como a quantidade de calorias, resultante dos alimentos elaborados (cardápio referência) para consumo durante a jornada de traba-
Iho. A partir de uma amostra do cardápio referência, procedeu-se as determinações:

1) das quantidades médias de alimentos consumidos per capita/dia; 2) da composição química desses alimentos ${ }^{25}$; 3) das quantidades médias de colesterole de ácidos graxos saturados, poliinsaturados e monoinsaturados, oferecidas/consumidas, na fábrica; 4) do consumo energético médio per capita/dia; e 5) da distribuição percentual média, per capita, dos nutrientes da refeição consumida na indústria independentemente do turno de trabalho. Os parâmetros para a alimentação/dia foram os da $\mathrm{OMS}^{20}$.

Para a análise, foram calculadas as prevalências, os IC a $95 \%$, a RP e o Odds Ratio $(O R)^{12,13}$. Posteriormente, as quotas energéticas médias diárias recomendadas - total e ocupacional - foram comparadas com a quota energética média oferecida, na fábrica, para consumo diário. Para identificação da variável modificadora de efeito, foi usado o teste da razão de verossimiIhança, mantendo-se, na análise a variável, caso a mesma apresentasse valor de $p$ $£ 0,05$. Para avaliar confundimento, adotou-se, como na avaliação de modificação de efeito, o procedimento backward. Seria mantida, como confundidora, a variável que apresentasse variação percentual da amplitude do intervalo de confiança da OR de, pelo menos, $15 \%$. O critério de inclusão, foi propositalmente, restrito.

\section{Resultados}

Dos 83 trabalhadores, $15(18,1 \%)$ tinham idade $<30$ anos, $44(53,0 \%) 30$ a 39 anos e $24(28,9 \%)=40$ anos, sendo $34(41 \%)$, em turno administrativo, e $49(59 \%)$, sob revezamento de turno, na área de produção. Pelo número de mulheres $(13,3 \%)$, os dados não foram estratificados por sexo. As prevalências das lípidas indesejáveis foram muito elevadas (Figura 1 e Tabela 1), sendo, a mais baixa, a das triglicérides, com $18,1 \%$.
* Programa implantado na fábrica, a partir de 1995, durante a avaliação da saúde do trabalhador, em cada exame periódico, com intuito de reduzir os riscos para a saúde, contidos na alimentação, para as doenças crônico-degenerativas. 
Figura 1 Prevalências do índice e dos níveis lipídicos indesejáveis, em trabalhadores de uma indústria petroquímica, PPC - BA, 1998.

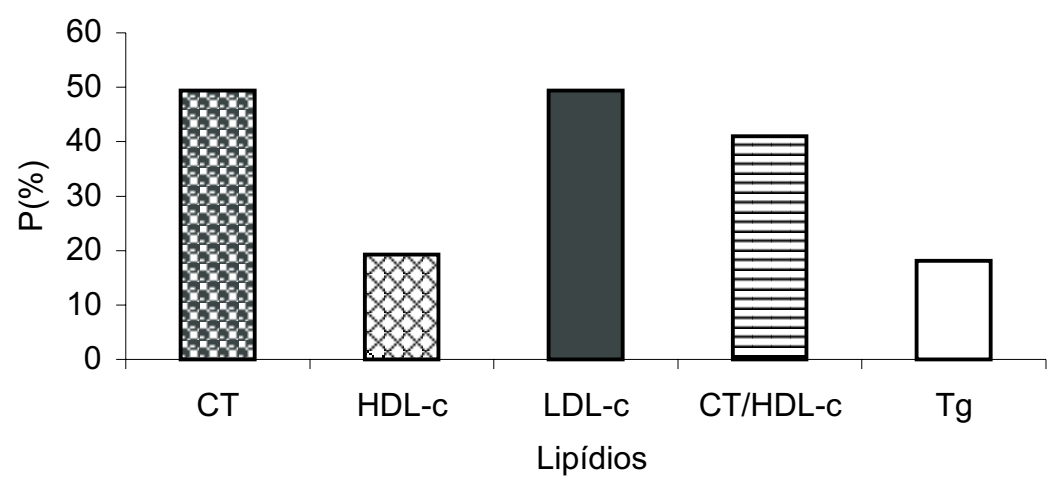

As prevalências de práticas alimentares, mais lipídicas, alcança $63,9 \%$ e não participação dos trabalhadores no PQV 60,2\% (Tabela 1).

\begin{tabular}{lllc}
\hline Variáveis & $\mathrm{n}^{\circ}$ & $\mathrm{P}, \%$ & $\mathrm{IC} 95 \%$ \\
\hline Práticas alimentares mais lipídicas & 53 & 63,9 & $(53,6-74,2)$ \\
Hipercolesterolemia & 41 & 49,4 & $(38,6-60,2)$ \\
HDL-C indesejável & 16 & 19,3 & $(10,8-27,8)$ \\
LDL-C indesejável & 41 & 49,4 & $(38,6-60,2)$ \\
CT/HDL-C indesejável & 34 & 41,0 & $(30,4-51,6)$ \\
Hipertrigliceridemia & 15 & 18,1 & $(9,8-26,4)$ \\
Programa de Qualidade de Vida - não participação & 50 & 60,2 & $(49,7-70,7$ \\
\hline
\end{tabular}

Perfil lipídico indesejável, foi observado em $81,1 \%$ dos trabalhadores que referiam consumir alimentação mais lipídica com RP de 1,43 , IC $(1,02 ; 2,01)$, em relação aos que consumiam uma alimentação menos lipídica. Essa associação foi positiva e es- tatisticamente significante (Tabela 2). Dos não participantes do PQV 76,0\% apresentam PLI contra $66,7 \%$ entre os que participavam do PQV. A RP foi 1,14 com IC 10,85 $-1,52)$, não estatisticamente significante.

Tabela 2 Associação entre perfil lipídico indesejável e dietas mais lipídicas e não participação do Programa de Qualidade de Vida, em uma indústria petroquímica, PPC BA, 1998.

\begin{tabular}{|c|c|c|c|c|c|c|c|}
\hline \multirow[t]{3}{*}{ Variáveis } & \multicolumn{4}{|c|}{ PLI } & \multirow{3}{*}{ Total } & \multirow{3}{*}{$\mathrm{RP}$} & \multirow{3}{*}{ IC 95\% } \\
\hline & \multicolumn{2}{|c|}{ Sim } & \multicolumn{2}{|c|}{ Não } & & & \\
\hline & $n^{\circ}$ & $\mathrm{P}, \%$ & $n^{\circ}$ & $\mathrm{P}, \%$ & & & \\
\hline \multicolumn{8}{|c|}{ Práticas alimentares ${ }^{*}$} \\
\hline Mais lipídicas & 43 & 81,1 & 10 & 18,9 & 53 & \multirow[b]{2}{*}{1,43} & \multirow[b]{2}{*}{$(1,02-2,01)$} \\
\hline Menos lipídicas & 17 & 56,7 & 13 & 43,3 & 30 & & \\
\hline \multicolumn{8}{|l|}{$\mathbf{P Q V}^{* *}$} \\
\hline Não participava & 38 & 76,0 & 12 & 24,0 & 50 & \multirow{2}{*}{1,14} & \multirow{2}{*}{$(0,85-1,52$} \\
\hline Participava & 22 & 66,7 & 11 & 33,3 & 33 & & \\
\hline
\end{tabular}

* Realizadas na indústria e fora dela.

** Programa de Qualidade de Vida (co-variável) realizado anualmente na indústria, a partir de 1995. 
A co-variável $P Q V$, apresentou p-valor igual a 0,14 , não sendo modificadora de efeito. Com a exclusão do PQV, não houve modificação relevante da variação da amplitude do intervalo de confiança da OR, não se detectando confundimento (Quadro 1).

Quadro 1 Avaliação de modificação de efeito e confundimento na associação entre práticas alimentares e perfil lipídico indesejável, a partir do programa de qualidade de vida.

\begin{tabular}{|l|c|c|c|c|c|c|c|c|}
\hline Modelo & $-2 \operatorname{Ln}(\mathrm{l})$ & $\begin{array}{c}\text { Teste Razão } \\
-2 \operatorname{Ln}(\mathrm{l})\end{array}$ & $\begin{array}{c}\text { Graus de } \\
\text { Liberdade }\end{array}$ & $\mathrm{X}^{2}$ & $\mathrm{p}$-valor & $\begin{array}{c}\text { Odds Ratio } \\
(\mathrm{OR})\end{array}$ & $\begin{array}{c}\text { Intervalo de } \\
\text { Confiança }\end{array}$ & $\begin{array}{c}\text { Amplitude do } \\
\text { intervalo }\end{array}$ \\
\hline Completo & 88,758 & & & & & 3,60 & $1,29-10,04$ & 8,75 \\
Reduzido & 90,907 & $90,907-88,758$ & 1 & 2,15 & 0,14 & 3,29 & $1,21-8,91$ & 7,70 \\
\hline
\end{tabular}

Todas as prevalências das lípidas indesejáveis, à exceção do HDL-c indesejável, aumentaram com a idade (Figura 2).

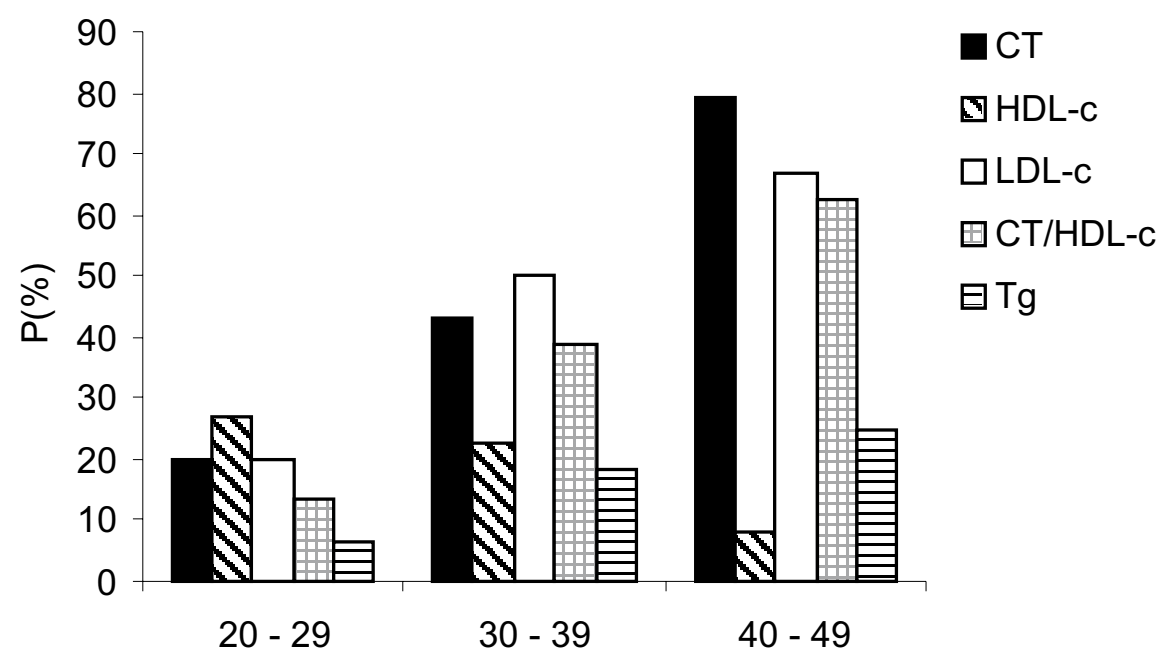

Faixa etária

Figura 2 Prevalências do índice e dos níveis lipídicos indesejáveis, por faixa etária, em trabalhadores de uma indústria petroquímica, PPC - BA, 1998.

Os percentuais referentes a QEO média, adequada aos trabalhadores, a QET e a QEF (Figura 3), permitem observar que a QEO correspondeu a aproximadamente, $40 \%$ da QET e que mesmo subtraindo o percentual da QEF em 1, 2 e até 3 desvios padrão desta média, continuam acima do recomendado e corresponde, a quase $100 \%$ da quota energética total média diária, recomendada para estes trabalhadores. 
${ }^{*}$ QET = Quota Energética Total média recomendada. QEO =

Quota Energética Ocupacional média recomendada. QEF = Quota Energética planejada/consumida na Fábrica.

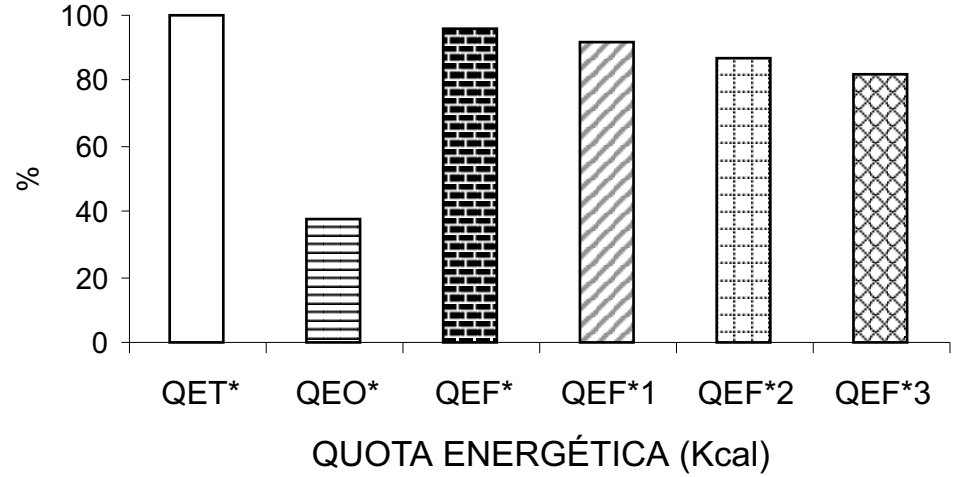

Figura 3 Percentuais das quotas energéticas médias recomendadas e da oferecida aos trabalhadores de uma indústria petroquímica, PPC - BA, 1998.

Na Figura 4, encontra-se a distribuição dos macronutrientes na refeição consumida durante a jornada de trabalho e aquelas que são recomendadas. A quota de glicídios encontra-se abaixo, a de proteínas, acima, e a lipídica, com valor no limite máximo do recomendado pela OMS, para uma refeição normocalórica.

${ }^{*}$ Rec. = Recomendado. Ind. Turno = Independentemente de Turno. $A D M=$ Turno Administrativo. RT = Revezamento de Turno.

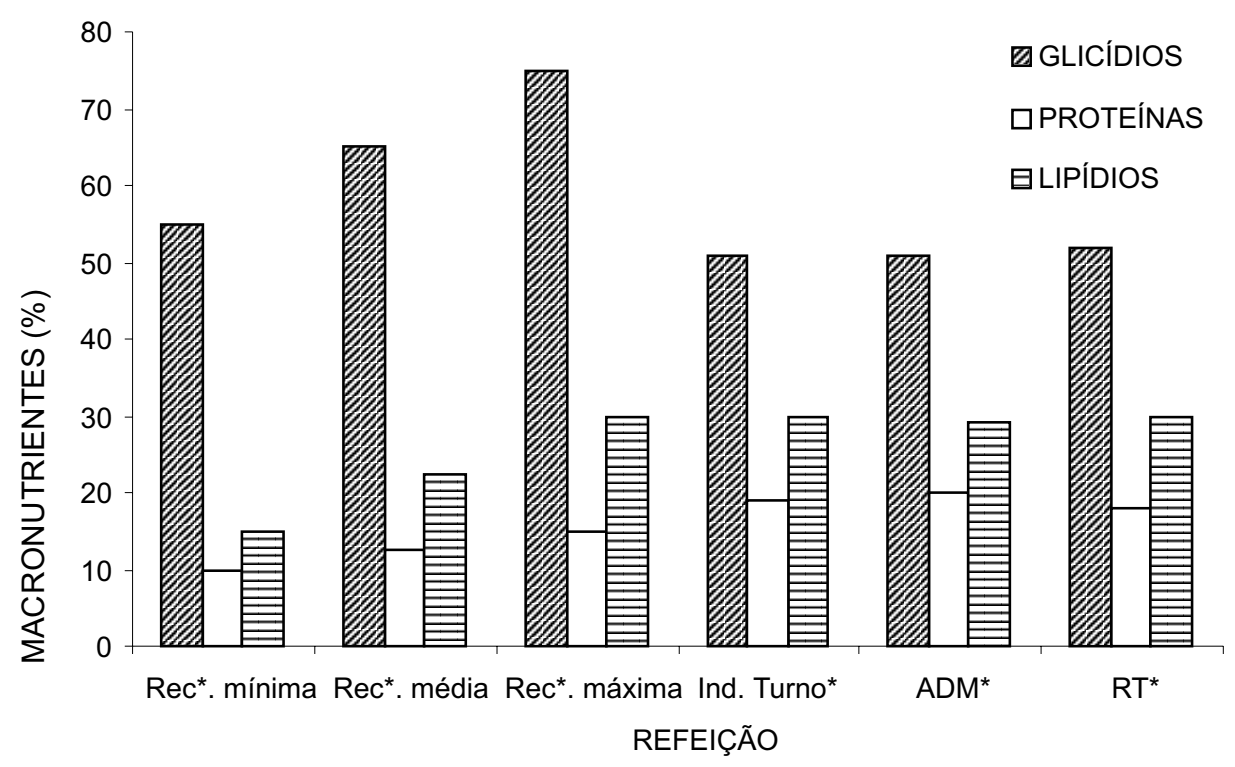

Figura 4 Distribuição dos macronutrientes na refeição recomendada e da consumida em uma indústria petroquímica, PPC - BA, 1998.

As proporções médias dos ácidos graxos saturados e poliinsaturados na refeição consumida, independentemente do turno (Figura 5), apresentam-se nos limites máximos recomendados. Nos trabalhadores no turno administrativo, a quantidade de ácidos graxos saturados foi de $14 \%$ e a consumida pelos trabalhadores, com revezamento de turno, apresenta uma quantidade de poliinsaturados de $8 \%$. 


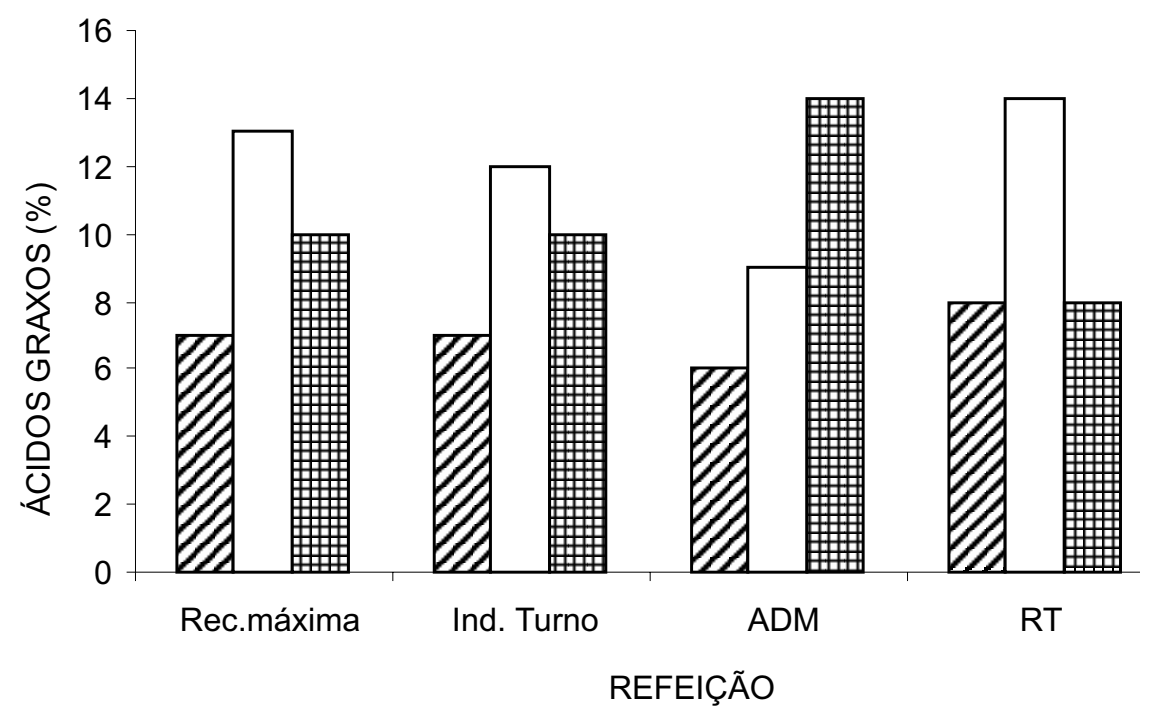

$\square$ Poliinsaturados $\square$ Monoinsaturados $⿴$ Saturados

Figura 5 Percentuais de ácidos graxos recomendados e consumidos na refeição em uma indústria petroquímica, PPC - BA, 1998.

Na Figura 6, consta uma comparação entre a consumida e a recomendada. A quantidade média do colesterol, consumida pelo trabalhador da indústria, mostra-se acima da máxima recomendada/dia.

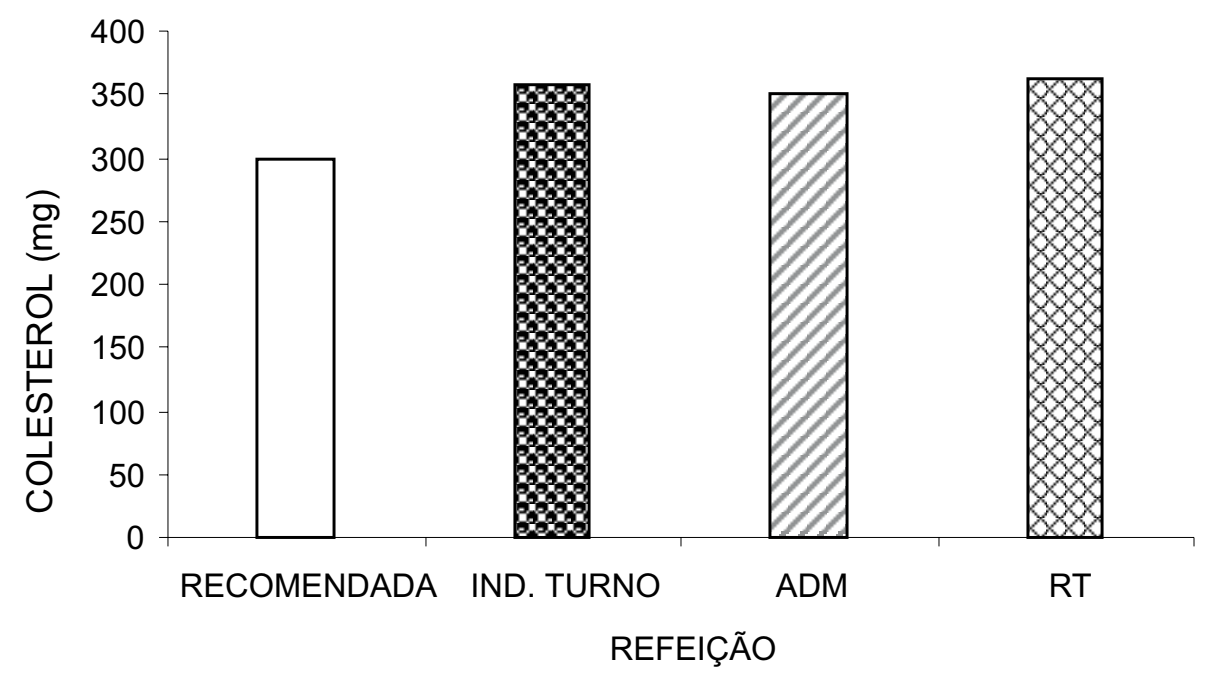

Figura 6 Quantidade de colesterol recomendada e consumida em média/dia em uma indústria petroquímica, PPC - BA, 1998. 


\section{Discussão}

Além da similaridade qualitativa, entre as refeições consumidas dentro e fora da indústria, o padrão alimentar dos trabalhadores mostrou-se, bastante próximo dos resultados dos inquéritos domiciliares realizados no Brasil em décadas passadas, quando se observaram tendências, na redução do consumo de cereais e tubérculos, da substituição dos glicídios por lipídios e da troca de proteínas vegetais por animais 8,10 . A refeição consumida na indústria, independentemente do turno de trabalho, contém alto teor de gordura, tendo como agravante, a qualidade dos lipídios consumidos. Apesar de a maior parte do colesterol sérico ser endógeno, a composição lipídica da alimentação exerce papel importante, na regulação do nível sérico de colesterol, uma vez que, o grau de saturação dos ácidos graxos exerce influência sobre os níveis de LDL-c e de HDL-c de forma diferenciada. De modo geral, os ácidos graxos saturados tendem a elevar tanto o LDL-c quanto $\circ \mathrm{HDL}-\mathrm{c}$, enquanto os poliinsaturados, quando usados em substituição aos saturados, reduzem, tanto o LDLc quanto o HDL-c ${ }^{15}$.

A queda da prevalência do HDL-c indesejável, a partir dos 30 anos de idade, acentuando-se ainda mais a partir da quarta década, difere da literatura. Segundo alguns estudos, o HDL-c, entre a terceira e a sexta décadas, mantém-se estável nos homens e, seguindo-se de pequena elevação, enquanto, nas mulheres, tende a elevar-se, lentamente, até a sétima década, com pequena redução posterior. Entretanto, as concentrações plasmáticas do HDL-c permanecem, significativamente mais elevadas, nas mulheres, do que nos homens ${ }^{6}$.

Ainda que não se possa afirmar que as alterações do perfil lipídico que ocorrem, com a idade, sejam devidas ao envelhecimento, a hábitos de vida, relacionados à dieta e níveis de atividade, ou uma combinação destes fatores, sabe-se que as práticas alimentares desempenham um importante papel, nestas alterações. Os dados mais convincentes de que a dieta se constitui num importante fator modulador no metabolismo das lipoproteínas, nas alterações relacionadas com a idade, são a ausên- cia de elevação no colesterol plasmático, nas comunidades, onde os indivíduos consomem dietas pobres em gorduras saturadas e colesterol ${ }^{6}$.

Investigações realizadas em grupos populacionais migrantes revelam a elevação dos níveis médios de CT e do LDL-C, quando, hábitos alimentares inadequados foram incorporados por estes grupos ${ }^{24}$. Outros estudos confirmam a associação positi$v a$, entre consumo de alimentos, fonte de proteína animal e de lipídios e hiperlipidemias ${ }^{15,19}$.

O aumento do nível sangüíneo de CT está associado ao consumo elevado de colesterol e ácidos graxos saturados, na dieta, e a uma baixa ingestão de ácidos graxos poliinsaturados ${ }^{28}$. Entretanto, Mensink \& Katan ${ }^{17}$ demonstraram, ser pouco relevante, o efeito dos ácidos graxos poliinsaturados sobre o nível de colesterol sangüíneo. Essa afirmação pode estar relacionada ao percentual, mais elevado de ácidos graxos poliinsaturados, em dietas contemporâneas (em torno de $6 \%$ da energia), do que as de, aproximadamente, $3 \%$, em dietas de décadas passadas ${ }^{17}$. Desse modo, podese suspeitar que as características qualitativas da refeição consumida diariamente, na indústria - alimentação com excesso de colesterol (aproximadamente $350 \mathrm{mg}$ ) e de ácidos graxos saturados $110 \%$ da quota energética já excessiva) -, é a mais provável explicação para a associação positiva, entre as práticas alimentares e o perfil lipídico, dos trabalhadores. Assim, são fortes os indícios de que a alimentação desses trabalhadores se constitui, num possível fator de risco, para o perfil lipídico indesejável.

Ainda que os mecanismos, pelos quais ocorrem as dislipidemias, não estejam totalmente esclarecidos, uma vez que, nem sempre, está presente em pessoas com sobrepeso e/ou distribuição andróide. Os resultados mostram-se coerentes com estudos, realizados em diferentes grupos populacionais ${ }^{16}$, nos quais foram descritas associações entre ingestão de ácidos graxos saturados e elevação do $C T$, em especial, o LDL-c. Outros estudos apontam a influência do colesterol dietético sobre o CT sérico, apesar da variabilidade, na magnitude da resposta ${ }^{24}$.

A elevada freqüência de trabalhadores que informou não participar do PQV 
demostra a falta de interesse pelo Programa, pouca informação sobre repercussões maléficas, do estilo de vida inadequado para a saúde, em particular, das práticas alimentares ou falha nas estratégias do Programa. Esse comportamento pode estar associado ao fato de eles rejeitarem a proposta do Programa, para mudanças das suas práticas alimentares, já asseguradas, uma vez que, só $39,8 \%$ aderiu ao PQV.

A associação borderline entre $P Q V$ e perfil lipídico indesejável, parece resultar da persistência de práticas alimentares inadequadas. $O$ fato de o PQV estabelecer modificações na composição de alguns itens do cardápio, visando redução de riscos para a saúde, provavelmente, ainda é insuficiente para essa mudança. $O$ argumento se fundamenta, no fato em que, uma dieta normocalórica, com ação preventiva, não pode restringir-se a mudanças da alimentação, apenas numa única refeição.

Se o objetivo principal do PQV é reduzir os riscos, para a saúde, contidos na dieta, para as doenças crônico-degenerativas, a participação da indústria, para prevenir tais agravos, mostrou-se pouco eficiente. Desse modo, a proposta do $P Q V$, em reduzir a quantidade de gordura e aumentar a de fibras, na refeição consumida na indústria, não previne os agravos em questão, principalmente, se considerarmos que a reformulação de práticas alimentares inadequadas não se constitui num processo de fácil mudança, a curto prazo. Ademais, as mudanças de comportamento, necessárias ao alcance do objetivo do PQV, não se concretizam com uma única intervenção anual.
Também para o $P Q V$, a sua não interferência na associação investigada orientou para a exclusão dessa variável do modelo, sem prejuízo, pois não se confirmou, como modificadora de efeito, ou como confundidora. Entretanto, não se pode excluir a possibilidade de que a não interferência da variável na associação investigada seja, também, atribuída à falta de poder do estudo, em função do tamanho reduzido da população.

Além do excesso de calorias, de gordura saturada e de colesterol, identificados na alimentação consumida, durante a jornada de trabalho, fora da indústria, esses trabalhadores, têm como agravante, os seus estilos de vida, mantendo um padrão de alimentação, com características semelhantes àquela ofertada e consumida na indústria, associado a uma atividade física, igualmente, insuficiente. Desse modo, as práticas alimentares dos trabalhadores, em particular, no local de trabalho, contribuem para a perpetuação de estilo de vida inadequado, evidenciado pelas dislipidemias. O programa de qualidade de vida da empresa, ainda é incipiente, e de curta duração para alcançar os objetivos a que se propõe.

Os resultados mostram, claramente, que a incorporação pelas indústrias dos mínimos calóricos recomendados pelo PAT, para a refeição, no local de trabalho, é incompatível com as necessidades nutricionais dos seus trabalhadores e promotora de riscos, para as maiores causas de morbidade e mortalidade, associadas à alimentação.

\section{Referências Bibliográficas}

\author{
1. ALMEIDA FILHO, N., ROUQUAYROL, \\ $M . Z$. Introdução à \\ epidemiologia moderna. 2.ed. \\ Rio de Janeiro: COOPEMED/ \\ APCE/ ABRASCO, 1992.
}
2. AUGUSTO, A. L. P. et al. Terapia nutricional. São Paulo: Atheneu, 1993.

3. BARRETO, M. L., CARMO, E. H. Tendências recentes das doenças crônicas no Brasil. In: LESSA, I. (Org.) $O$ adulto brasileiro e as doenças da modernidade: epidemiologia das doenças crônicas não-transmissíveis. São Paulo: Hucitec, 1998. p. 15-27. 
4. BRASIL. Ministério do Trabalho. Programa de Alimentação do Trabalhador - PAT. Manual de Legislação. Brasilia: Secretaria de Segurança e Saúde no Trabalho, 1997.

5. EPSTEIN, F. H., HIGGINS, $M$. Epidemiology of obesity. In: BJÖRNTORP, P. BRODOFF, B. N. Obesity. Philadelphia: J. B. Lippincott Company, 1992. v.1 chap. 27 , p. $330-342$.

6. ETTINGER, W. H. Níveis lipídicos e risco de cardiopaita coronariana aterosclerótica entre idosos. In: BENDER, S. B., CARANASOS, G. J. (Orgs.) Clínicas médicas da América do Norte. Rio de Janeiro: Interlivros, 1989. v. 6, p. 1.669 1.674

7. FONSECA, M. H. G. A. Epidemiologia da obesidade em adultos normoglicêmicos:estudo em cinco macroregiões brasileiras. Tese. Rio de Janeiro - Rio de Janeiro. (Doutorado em Medicina) - Faculdade de Medicina da Universidade Federal do Rio de Janeiro, 1997.

8. FUNDACÃO GETÚLIO VARGAS. U.S. DEPARTMENT OF AGRICULTURE, ECONOMIC RESEARCH SERVICE. Food consumption in Brazil: family budget survey in the early 1960's. Jerusalem: Keter Press, 1970.

9. GORENDER, J. Globalização, tecnologia e relações de trabalho. Estudos Avançados, v. 29, n. 11 , p. 311-361, 1997.

10. IBGE. Pesquisa de orçamento familiar - 1987/1988. Rio de Janeiro, 1991. v.1.

11. KISSEBAH, A. H. et al. Obesidade e riscos de saúde. In: BRAY, G. A. (Org.) Clínicas médicas da América do Norte. Rio de Janeiro: Interlivros, 1989. v.1, p.131-162.
12. KLEINBAUM, D. G. Logistic regression: a self-learning text. New York: Springer, 1992.

13. KLEINBAUM, D. G. et al. Applied regression analysis and other multivariables methods. 2.ed. Belmont, California: Duxbury Press, 1998.

14. LESSA, I. Doenças crônicas nãotransmissíveis. In: (Org.) 0 adulto brasileiro e as doenças da modernidade: epidemiologia das doenças crônicas nãotransmissíveis. São Paulo: Hucitec, 1998. p. 29-42.

15. MAHAN, L. K., ARLIN, M. T. Krause: alimentos, nutrição e dietoterapia. 8. ed. São Paulo: Roca, 1995.

16. MARTINEZ, T. L. R. Condutas clínicas nas dislipidemias. Belo Horizonte: Saúde, 1997.

17. MENSINK, R. P., KATAN, M. B. Effect of dietary fatty acids on serum lipids and lipoproteins: a meta-analysis of 27 trials. Arterioscler Thromb, n.12, p. 911-919, 1992.

18. MONDINI, L., MONTEIRO, C. A. Mudanças no padrão de alimentação. In: MONTEIRO, C. A. (Org.) Velhos e novos males da saúde no Brasil: a evolução do País e de suas doenças. São Paulo: Hucitec, 1995. P. 79-89.

19. NEVES, N. M. de S. Nutrição e doença cardiovascular. Rio de Janeiro: Guanabara, 1997.

20. OMS. Grupo de Estudio. Dieta, nutrición y prevención de enfermedades crónicas. Ginebra, 1990 (Serie de Informes Técnicos).

21. OMS. Grupo de Estudos. Necessidades de energia e proteína. São Paulo: Roca, 1998 (Série de Relatos Técnicos). 
22. OMS. Reuniones sobre nutrición. Necesidades de energía y de proteínas. Ginebra, 1973 (Serie de Informes Técnicos).

23. PALLOIX, C. O processo de trabalho: do fordismo ao neofordismo. In: ERBER, F. S. (Org.) Processo de trabalho e estratégias de classe. Rio de Janeiro: Zahar, 1982.

24. SOCIEDADE BRASILEIRA DE CARDIOLOGIA - SBC. $2^{\circ}$ Consenso brasileiro sobre dislipidemias: detecção, avaliação e tratamento. Arq. Bras. Cardiol., v. 67, p.116, 1996.

25. UNIVERSIDADE FEDERAL DE SÃO PAU-
LO - UFSP. Escola Paulista de Medicina. Programa de Apoio à Nutrição versão 2.5. São Paulo, 1995.

26. VIANA, S. V. Nutrição, trabalho \& sociedade. Rio de Janeiro: HUCITEC, 1996.

27. WHO. Study Group. Diet, Nutrition and Prevention of Chronic Diseases. Geneva, 1990 (Technical Report Series).

28. WILLETT, W. Diet and coronary heart disease. In: Nutritional epidemiology. 2.ed. New York: Oxford University Press, 1998. p. 414466. 